EDITORIAL

\title{
Spread through airspaces (STAS) on frozens: too much,
} too soon

(c) The Author(s), under exclusive licence to United States \& Canadian Academy of Pathology 2021

Modern Pathology (2022) 35:140-141; https://doi.org/10.1038/s41379021-00957-w

Imagine you are the pathologist covering frozen sections today. Your surgeon comes down with a lung wedge and says to you "Dr. X, this patient has a lung adenocarcinoma. I need to know whether there's STAS". You scramble to look up what STAS is and find out that it means "spread through airspaces", and that you should find tumor cells inside the alveoli beyond the main tumor. You panic. How do they know that's not some kind of artefact? You used to ignore those cells as floaters when you were a resident! More importantly, how will you know they are not an artefact on a frozen section slide? You are not a lung pathologist and you've never encountered this issue at frozen section before. You call your surgeon over the phone. "Dr. Y, will this change your management?" The surgeon replies "Yes. If you tell me there is STAS, I will proceed to lobectomy. If not, l'll stop now and close up". You wipe a bead of sweat from your brow and stare at the slide.

You call it STAS on frozen, the lobe comes out, and on permanents your colleague says she cannot find any STAS. Now you will be known as the pathologist responsible for a patient unnecessarily losing a lobe of lung because you over-called STAS on frozen section! Will your surgeon ever trust you again? And most importantly, how will the patient feel to have lost a lung lobe for no good reason when they could have had a smaller surgery? Now play this scenario over and over around scores of departments around the country.

In this month's issue, Zhou et al. address this matter head on ${ }^{1}$. They ask the question: can we accurately determine whether there is STAS on frozen sections, and should that be the sole basis to proceed to lobectomy in patients who would otherwise be treated with a sublobar resection? In a well-conducted study of 163 stage I lung adenocarcinomas, the authors show that even in the hands of expert pulmonary pathologists, STAS is both overdiagnosed and underdiagnosed at frozen section at unacceptably high rates. Frozen sections miss nearly half of all cases of true STAS (sensitivity: 55\%), and $20 \%$ of all cases called STAS at frozen section turn out to be incorrect on permanents (specificity: $80 \%$ ). Most importantly, the authors show that if STAS were used as an indication to proceed to lobectomy, overdiagnosis of STAS at frozen section would result in unnecessary lobectomy in 13 of 163 cases (8\%). Zhou et al. also suggest that determination of tumor grade at frozen section might be a better parameter than STAS in deciding whether to upgrade to lobectomy, an observation that needs to be tested by other groups in future studies. These and other studies highlight the difficulties of using frozen sections to assess histologic parameters that are typically assessed on permanent sections in lung cancer ${ }^{2,3}$.

The concept of STAS is quite recent but the buzz around it has grown rapidly within pulmonary pathology circles. On permanent sections, STAS has been associated with an increased likelihood of lymph node metastases and aggressive behavior not just in adenocarcinoma but in a host of other lung neoplasms. It has even been introduced as a reportable element in College of American Pathologists lung cancer synoptic templates. The concept has ardent supporters and vigorous detractors. Supporters claim that it is a finding of great prognostic import ${ }^{4,5}$, while detractors posit that STAS is an artefact ${ }^{6}$. Yet others feel that both STAS and morphologically similar artefacts exist, but emphasize that it could be challenging to tell them apart, in particular, on frozen section ${ }^{7}$. While this debate continues, the rest of the pathology world watches curiously and with measured skepticism.

A study suggesting that STAS could guide surgeons regarding which lung adenocarcinoma patients to take to lobectomy versus which ones to stop at a wedge or other sublobar resection came to the notice of surgeons around the country, and pathologists began to be asked whether they were prepared to diagnose STAS on frozen section ${ }^{8}$. As pathologists scrambled to see how robust the literature on this subject was, it was apparent that there was very little published literature on this issue; the few studies that did exist raised concerns regarding the use of frozen sections for detecting STAS $^{7,9}$. In this environment, a study soberly examining the nitty-gritty of this subject was sorely needed, and we now have some hard data to discuss. The crux of the issue is this: are surgeons willing to perform unnecessary lobectomies on $8 \%$ of their patients based on an over-call of STAS at frozen section? The reader is reminded that this study was performed in an academic center by pulmonary pathology subspecialists. Away from the ivory towers of academia, in the hands of general surgical pathologists, this percentage is likely to be much higher.

The underlying issue is bigger than that of academia vs. community practice. It concerns the need to determine which histologic parameters (if any) can be assessed on frozen section with a high enough sensitivity and specificity to aid surgeons in the difficult decision of whether to perform a sublobar resection or lobectomy in a patient with lung cancer. The indications that prompt this decision are currently evolving; other than the patient's ability to tolerate lobectomy, features that currently prompt consideration of sublobar resection include small size $(<2 \mathrm{~cm})$, slow growth rate and predominant ground-glass appearance on imaging.

The goal of pathologists helping surgeons to make a decision on limited resection vs. lobectomy is laudable, and the topic is worth studying. If pathologists can help in this decision, this would be a "win-win" situation. However, the study conducted by Zhou et al., and other published studies on this subject, strongly suggest that surgeons should not regard STAS on frozen section as the sole basis for converting a sublobar resection to a lobectomy.

There will no doubt be other studies on this issue, debate will ensue, and sides will be taken. That is how the process should work. We should have data, followed by more data, followed by vigorous debate, followed by a decision on whether established practice should be changed. What is dangerous is changing established practice on the basis of inadequate data, only to unleash catastrophe on patients. 
Time will tell whether the concept of STAS finds widespread acceptance and survives the test of time. For now, expecting pathologists to diagnose this finding on frozen section and bear responsibility for an aggressive surgical resection is fraught with risk. It is too much, too soon.

Sanjay Mukhopadhyay ${ }^{1 \times}$ and Monisha Sudarshan ${ }^{2}$ ${ }^{1}$ Department of Pathology, Cleveland Clinic, Cleveland, $\mathrm{OH}$, USA. ${ }^{2}$ Department of Thoracic and Cardiovascular Surgery, Cleveland Clinic, Cleveland, OH, USA. ${ }^{\circledR e m a i l: M U K H O P S @ c c f . o r g ~}$

\section{REFERENCES}

1. Zhou, F. et al. Assessment of the feasibility of frozen sections for the detection of spread through air spaces (STAS) in pulmonary adenocarcinoma. Mod Pathol. (2021). online ahead of print.

2. Trejo-Bittar, H. E., Incharoen, P., Althouse, A. D. \& Dacic, S. Accuracy of the IASLC/ ATS/ERS histological subtyping of stage I adenocarcinoma on intraoperative frozen sections. Mod. Pathol. 28, 1058-1063 (2015).

3. Yeh, Y. C. et al. Using frozen section to identify histological patterns in stage I lung adenocarcinoma of $\leq 3 \mathrm{~cm}$ : accuracy and interobserver agreement. Histopathology 66, 922-938 (2015).

4. Kadota, K. et al. Tumor spread through air spaces is an important pattern of invasion and impacts the frequency and location of recurrences after limited resection for small stage I lung adenocarcinomas. J. Thorac. Oncol. 10, 806-814 (2015).

5. Warth, A. Spread through air spaces (STAS): a comprehensive update. Transl. Lung Cancer Res. 6, 501-507 (2017).

6. Blaauwgeers, H., Russell, P. A., Jones, K. D., Radonic, T. \& Thunnissen, E. Pulmonary loose tumor tissue fragments and spread through air spaces (STAS): invasive pattern or artifact? A critical review. Lung Cancer 123, 107-111 (2018).
7. Villalba, J. A. et al. Accuracy and reproducibility of intraoperative assessment on tumor spread through air spaces in stage 1 lung adenocarcinomas. Mod. Pathol. 16, 619-629 (2021).

8. Eguchi, T. et al. Lobectomy is associated with better outcomes than sublobar resection in spread through air spaces (STAS)-positive T1 lung adenocarcinoma: a propensity score-matched analysis. J. Thorac. Oncol. 14, 87-98 (2019).

9. Walts, A. E. \& Marchevsky, A. M. Current evidence does not warrant frozen section evaluation for the presence of tumor spread through alveolar spaces. Arch. Pathol. Lab. Med. 142, 59-63 (2018).

\section{AUTHOR CONTRIBUTIONS}

Both authors contributed to the drafting and approval of this manuscript, and take responsibility for its contents.

\section{COMPETING INTEREST}

The authors declare no competing interests.

\section{ADDITIONAL INFORMATION}

Correspondence and requests for materials should be addressed to Sanjay Mukhopadhyay.

Reprints and permission information is available at http://www.nature.com/ reprints

Publisher's note Springer Nature remains neutral with regard to jurisdictional claims in published maps and institutional affiliations. 